\title{
Texture Analysis using Rough Texton
}

\author{
Phani Kumar Talluri \\ Computer Science and Engineering \\ Vasireddy Venkatadri Institute of Technology \\ Guntur, A.P., India
}

\author{
Sudhakar Putheti \\ Computer Science and Engineering \\ Vasireddy Venkatadri Institute of Technology \\ Guntur, A.P., India
}

\begin{abstract}
The present paper derived a new texture classification tactic using textons and rough sets. Texton is a statistical approach used to analyze the texture of an image. Textures will be developed only if the side elements lie within the contiguity. Texton Image has the discrimination power of color, texture and shape features. In the proposed tactic texton using rough texture spectrum and color features are calculated using HSV color space. Rough texture spectrum covers the entire range that is difficult unless otherwise. Rough set theory is better handles vagueness. The designed mechanism is estimating interesting as it enumerates contrasting visage with confined number of preferred components. The experimental results suggest the adequacy of the present mechanism over the various other approaches.
\end{abstract}

\section{Keywords}

Texton; Rough Sets; Rough Texture Unit; Texture Classification; Texture Unit;

\section{INTRODUCTION}

Texture is one of the most important means to classify images. Texture analysis is used to group the different regions in same image. Texture analysis is also used in finger prints analysis in foreign seek labs. Texture analysis refers to the section of imaging science that is bothered with the depiction of peculiar image properties by textural features. Nonetheless, there is by no means cosmically acknowledgement interpretation of what image pattern is and in matter of course distinctive experimenter use divergent annotations confined in the itemized sphere of appliance [1]. The essential image handling regulations in which pattern scrutiny approaches are used are codification, demarcation and amalgamation. In image codification the intent is to assort distinct images or image domains into definite troops [2]. Pattern scrutiny mechanisms are pertinent to this because they implement solitary evaluation on the pattern, or dimensional variation of pixels, of the region where they are applied. In image demarcation predicaments the plan is to enact circumscription among different image domains [3]. By practicing texture scrutiny techniques to an image, and deceiving the explicit locale where texture feature values change significantly, boundaries between regions can be established.

For diagnostics and therapy in medical field, use of digital images is essential and it became a fundamental component for diagnosis [4], treatment planning [5], and assessing response to treatment [6]. By using texture classification tactics it is Possible to retrieve relevant images which are help for diagnose the health Problem.

The ease of the paper is formulated as follows. Existing texture scrutinity tactics and rough sets are discussed in section 2. Our proposed tactic for texture classification of images is explained in section 3. Section 4 gives the details of the experiments followed by the results.

\section{EXISTING SYSTEM}

In medicine, pulmonologists, who use automated computer assisted diagnosis, requires images which are close to given image.

\subsection{Texture Scrutinity Tactics}

Existing texture scrutiny tactics are broadly divided into two categories: statistical tactics and structural tactics in most of the surveys [7-14]. But in the modern writings, various standard placed tactics have been employed in framework scrutiny [15], made up of auto inverted mechanism, Gaussian Markov indiscriminate portraits, Gibbs random portraits, Wold portrait, wavelet portrait, multichannel Gabor portrait and steerable pyramid, etc. These portraits present added effectual mechanisms for invariable structure scrutinity. Since they have various homogenous properties, these tactics analyzed into a comparably autonomous section to extant a pleasant analysis.

Previously for achieving better classification on texture scrutinity, the two important supervised classification tactics used are Texture Spectrum Operator (TSO) and Local Binary Pattern Operator (LBP). Approaches to texture scrutinity are usually categorized into structural, Statistical, Portrait-based and Transform.

\subsubsection{Structural Method}

Structural ways produce pattern by well-defined fundamentals (micro texture) and a pyramid of contiguous layouts (macro texture) of those fundamentals [11]. To describe the texture, one must define the fundamentals and the induction models. The advantage of the structural approach is that it presents an admirable significant depiction of the image. However, this feature is more useful for integration than scrutinity tasks. A powerful job for structural texture scrutinity is presented by mathematical morphology. It may prove to be useful for bone image scrutinity, e.g. for the detection of changes in bone microstructure.

\subsubsection{Statistical Method}

In statistical tactics, texture is described by a collection of statistics of selected features. Many of these tactics are stationed on Julesz's finding $[16,17]$ that the human perceptible system needs statistic appearances for texture prejudice counting the first-order statistics, the second- order statistics and the higher-order statistics. Accordingly, the statistics are broadly classified into first-order statistics, second-order statistics, and higher-order statistics. The basic variation is that first-order statistics evaluate properties (e.g. average and variance) of specific pixel standards, ignoring the contiguous intercommunication between image picture elements, whereas second- and higher order statistics evaluates premises of two or more element standards obtaining at definite sections referring to one another. 
Statistical tactics evaluate the contiguous diffusion of gray values, by measuring local features at each point in the image, and formulating a set of figures from the partitioning of the local features [18]. The logic behind this is the fact that the spatial division of gray values is one of the designating qualities of structure.

\subsubsection{Model-Based Method}

Earlier attention in texture analysis is mainly focused on the Orst- and second-order statistics of textures. Later, many model-based methods (including Gaussian Markov random fields [19], Gibbs random fields [20], and Wold model [21, 22]) are introduced to model texture. In these methods, a pattern image is shaped as a likeliness miniature or as a straightaway unification of a scheduled of basis functions. The coetaneous of these layouts are used to characterize feature images. The key problem of these methods is how to estimate the coefficients of these models and how to choose the correct model suitable for the selected texture. The coefficients of these models are often transformed into different forms invariant to translation, rotation and scale.

\subsubsection{Transform Model}

Alter tactics of texture scrutiny, for instance Fourier, Gabor and wavelet mutates stand for an image in an arena whose correlative entity has a perception that is meticulously relevant to the peculiarity of a pattern (such as prevalence or stature). Proceedings stationed on the Fourier transform percolate ailing in proceeding, by virtue of its deficit of dimensional boundaries. Gabor percolates furnish equipment for sophisticated dimensional boundaries; in spite of, their utility is restrained in habitude on the grounds that there is consistently no peerless percolate verdict at which one can restrain a dimensional architecture in habitual pattern. Correlated with the Gabor transform, the wavelet transforms element considerable eminence: changeable the dimensional verdict let on it to epitomize patterns at the utmost handy range, there is a ample dimension of distinction for the wavelet function, so one is able to co-opt wavelets finest befitting for pattern scrutiny in a exact operation.

\subsection{Rough Sets}

Rough set theory is still another way to ambiguity [23]. Equivalently to fuzzy set theory it is to classical set theory but it is encapsulated in it. Rough set theory can be noticed as a distinct application of Frege's idea of ambiguousness, i.e., unreliability in this path is shown by a edge region of a set, and not by a sectional membership, like in fuzzy set theory. Rough set theory can be explained quite generally by means of topological operations, interior and closure, called resemblances. Let us distinguish this complication more accurately. Presume given a set of objects $P$ called the universe and an un-deniability relation $\mathrm{Q} \subseteq \mathrm{P} \times \mathrm{P}$, symbolizing our lack of proficiency about elements of P. For the sake of uniformity estimated that $\mathrm{Q}$ is an equivalence relation. Let $\mathrm{A}$ be a subset of $\mathrm{P}$ to differentiate the set $\mathrm{A}$ with respect to $\mathrm{Q}$. To this end the basic concept of rough set theory given below.

The diminished similarity of a set A with respect to $\mathrm{Q}$ is the set of all objects, which can be for certain classified as A with respect to Q (are certainly with respect to $\mathrm{P}$ ) The loftier nearness of a set $A$ with respect to $R$ is the set of all objects which can be probably classified as A with respect to Q. The ending region of a set $\mathrm{A}$ with respect to $\mathrm{P}$ is the set of all objects, which can be classified neither as A nor as not-A with respect to $P$.
Set A is crisp (exact with respect to $\mathrm{Q}$ ), if the boundary region of A is empty. Set A is rough (inexact with respect to $P$ ), if the boundary region of $\mathrm{A}$ is nonempty. Rough sets can be also defined using a rough membership function, defined as

$\mu_{r}^{D}(r)=\frac{|A \cap D(r)|}{|D(r)|}$

Obviously

$\mu_{r}^{D}(r) \in[0,1]$

Value of the membership function $\mu_{\mathrm{r}}(\mathrm{A})$ is a kind of confining probability, and can be made clear as a degree of definiteness to which $r$ belongs to A (or $1-\mu_{\mathrm{r}}(\mathrm{A})$ as a degree of unpredictability.

Value of the membership function $\mu_{\mathrm{X}}(\mathrm{X})$ is a kind of conditional probability, and can be made clear as a degree of certainty to which $\mathrm{x}$ belongs to $\mathrm{X}$ (or $1-\mu_{\mathrm{X}}(\mathrm{X})$, as a degree of uncertainty).

The rough membership function can be used to define approximations and the boundary region of a set, as shown below:

$$
\begin{aligned}
& D_{*}(A)=\left\{r \in P: \mu_{A}^{D}(r)=1\right\} \\
& D^{*}(A)=\left\{r \in P: \mu_{A}^{D}(r)>0\right\} \\
& D T_{D}(A)=\left\{r \in P: 0<\mu_{A}^{D}(r)<1\right\}
\end{aligned}
$$

The rough membership function has the following properties [6-13]:

$$
\begin{aligned}
& \mu_{A}^{D}(r)=1 \text { iff } D_{*}(A) \\
& \mu_{A}^{D}(r)=0 \text { iff } r \in-D^{*}(A) \\
& 0<\mu_{A}^{D}(r)<1 \text { iff } x \in D T_{D}(A) \\
& \qquad \text { if } J(D)=\{(r, r): r \in P\}, \text { then }
\end{aligned}
$$

$\mu_{A}^{D}(r)$ is the characteristic function of $A$

$$
\begin{aligned}
& \text { if } r J(D) s, \text { then } \mu_{A}^{D}(r)=\mu_{A}^{D}(s) \text { provided } J(D) \\
& \mu_{P-A}^{D}(r)=1-\mu_{A}^{D}(r) \text { for any } r \in P \\
& \mu_{A \cup \gamma}^{D}(r) \geq \max \left(\mu_{A}^{D}(r), \mu_{\gamma}^{D}(r)\right) \text { for any } r \in P \\
& \mu_{A \cap \gamma}^{D}(r) \leq \min \left(\mu_{A}^{D}(r), \mu_{\gamma}^{D}(r)\right) \text { for any } r \in P
\end{aligned}
$$

The beyond holdings view absolutely the distinct fuzzy and rough membership. In peculiar properties (12) and (13) show that the rough membership precisely can be noticed as a statement of fuzzy membership. Let us recollect that the "rough membership", in contrast to the "fuzzy membership", has probabilistic flavor.

It can be easily seen that there exists a strict connection between ambiguity and contingency. As discussed above ambiguity corresponds to sets (concepts), whereas contingency is corresponds to objects of sets. Rough set approach shows clear connection between these two concepts. 


\section{PROPOSED SYSTEM}

Texture appears to differentiate between normal and abnormal pathologies. Our designed functioning has four different phases. They are

1. Division of color image into HSV planes.

2. Separation of Texton pictures from these color planes.

3. Abstraction of Texels (feature vectors) from these texton images.

4. Classification of Images

\subsection{Decomposition of Color Image into HSV Planes}

Color vision can be processed using RGB color space or HSV color area. RGB color space illustrates colors in premises of the amount of red, green, and blue present. HSV color space illuminates colors in provisions of the Hue, Saturation, and Value. In positions where color specification plays an constituent part, the HSV color portrait is often taken over the RGB model. The HSV modelelucidate colors correspondingly to how the human eye points to identify color. RGB describes color in terms of a combination of primitive colors, whereas, HSV illustrates color using more frequent observations such as color, vibrancy and brightness. The basketball robot uses HSV color space to process color vision.

\subsection{Texton Extraction}

In this proposed system rough texton approach is used to extract the texture. As per Julesz [16, 17] specification a texton is a arrangement which is shared by an image as a regular property. Patterns will be framed only if the neighboring components lie within the territory. Texton Image has the discrimination power of color, texture and shape features. Based on the Julez's $[16,17]$ textons theory the texton co-occurrence matrices (TCM) algorithm, can elucidate the structural correlation of textons for image retrieval. With the use of finite count of selected pixels the algorithm computes different features. Retrieval efficiency in CBIR will be increased by calculating texton using Rough texture spectrum and color features are calculated using HSV color space. Compared to RGB, HSV has its advantages. When turned as a shape description, texton deals straightly with familiar images and any distribution or object abstraction preprocessing stage will not be done. As a texture descriptor, good retrieval accuracy can be achieved especially for directional textures.

The texton can be inclined with the use of critical distances between texture elements. Texture element size determines the critical distance. Texture can be analyzed into minute units, like orientation, Texton classes of colors, extended blobs of specific width, aspect ratios and denominators of extended blobs. If texture elements are expanded to a large extent in one direction discrimination reduces. If the extended elements are not twiddled in position texture gradients increase at boundaries. Hence by using a sub image of size $3 \mathrm{x}$ 3 a texton gradient can be obtained. Here 12 textons of $3 \times 3$ grids are proposed as shown in figure 1. Even the reoccurrence chance of similar valued pixels in $3 \times 3$ grids is lesser than that of $2 \times 2$ grid, the textons refined using $2 \times 2$ grid may not give intact information about direction. The computational multiplicity for using the overlapped segments of 12 textons is also less to obtain final texton image. The 12 textons of $3 \times 3$ grid is used in the proposed tactic [24].

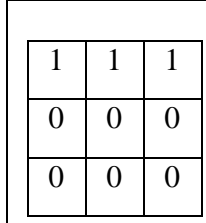

(a)

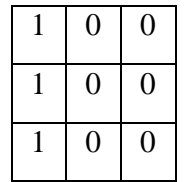

(d)

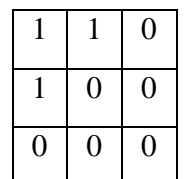

(g)

\begin{tabular}{|c|c|c|}
\hline 0 & 0 & 0 \\
\hline 1 & 0 & 0 \\
\hline 1 & 1 & 0 \\
\hline
\end{tabular}

(j)

\section{Fig 1: 12 types 0}

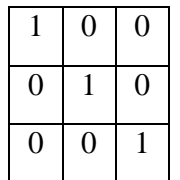

(k)

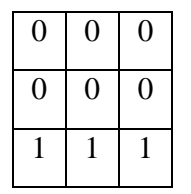

(c)

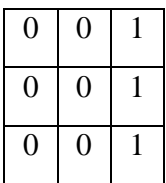

(f)

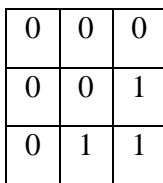

(i)

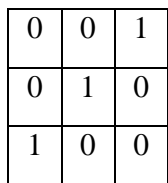

(1)
Determination of texture property can be done based on the values of Texture Unit and Texture Unit Number of an image plane [24]. A $3 \times 3$ pixel square will be constructed to define a basic unit of the tactic. $3 \times 3$ pixel square consists of a central pixel surrounded by eight neighbors. The square image has the information regarding regional texture in all directions of the central pixel.

Extraction of information regarding local texture of the surrounding pixels is the main objective. The size of the block is $3 \times 3$ pixels in present case. The nine pixels of this $3 \times 3$ square pattern of the image is indicated $\mathrm{Z}$, where $\mathrm{Z}$ consists of nine elements as $\mathrm{Z}=\left\{\mathrm{Z}_{0}, \mathrm{Z}_{1}, \mathrm{Z}_{2}, \ldots, \mathrm{Z}_{8}\right\}$, where $\mathrm{Z}_{0}$ corresponds to the central pixel's intensity and $\mathrm{Z}_{\mathrm{i}}(1 \leq \mathrm{i} \leq 8)$ corresponds to intensity surrounding pixel. Texture Unit (TU) is nothing but the unit which best suits for the confined texture component of a given pixel and its neighborhoods, in all directions of a square raster. Texture Unit (TU) as in [24], will be given by $\mathrm{TU}=\left\{\mathrm{F}_{1}, \mathrm{~F}_{2}, \mathrm{~F}_{3}, \ldots, \mathrm{F}_{8}\right\}$, where:

$$
\mathrm{F}_{\mathrm{i}}=\left\{\begin{array}{c}
0 \text { if } \mathrm{Z}_{\mathrm{i}}<\mathrm{Z}_{0} \text { and } \mathrm{Z}_{\mathrm{i}}<\mathrm{p} \\
1 \text { if } \mathrm{Z}_{\mathrm{i}}<\mathrm{Z}_{0} \text { and } \mathrm{a}_{\mathrm{i}}>\mathrm{p} \\
2 \quad \text { if } \mathrm{Z}_{\mathrm{i}}=\mathrm{Z}_{0} ; 1 \leq \mathrm{i} \leq 8 \\
3 \text { if } \mathrm{Z}_{\mathrm{i}}>\mathrm{Z}_{0} \text { and } \mathrm{Z}_{\mathrm{i}}<\mathrm{q} \\
4 \text { if } \mathrm{Z}_{\mathrm{i}}>\mathrm{Z}_{0} \text { andZ } \mathrm{an}_{\mathrm{i}}>\mathrm{q}
\end{array}\right.
$$

where predefined or user defined values will be assigned to $p$ and $\mathrm{q}$. And element $\mathrm{F}_{\mathrm{i}}$ engross the same position as occupied by pixel $\mathrm{i}$.

\begin{tabular}{|c|c|c|}
\hline 221 & 130 & 167 \\
\hline 125 & 155 & 52 \\
\hline 241 & 207 & 105 \\
\hline
\end{tabular}

(a)

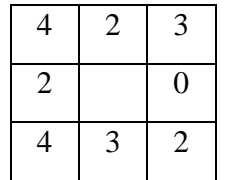

(b)
Fig. 2: (a) Hue levels of an image part. (b) Texture Unit associated to the central pixel. 
The total number of TU is 390625 (58). That can be obtained that each element of the TU can have any one of three desirable values $0,1,2,3$ or 4 . TU can be identified and can be organized in different ways; each TU will be labeled as a 5base number, named Texture Unit Number, NTU will be calculated by suing the formula:

$M_{T U}=\sum_{i=1}^{8} F_{i} \cdot 5^{\frac{i-1}{2}}$

Where $\mathrm{i}$ denote position of Texture Unit box, and Fi corresponds to value of the box $(0,1,2,3$ or 4$)$. The 8 elements can be organized individually. As shown in figure 4 where the elements are ordered in clock wise direction, the first element can have eight possible positions, from ' $a$ ' to ' $h$ ' in clockwise order and then the 16777216 texture units can be classified by the abode formula under eight different ordering ways (from a to $h$ ).

\begin{tabular}{|l|l|l|}
\hline $\mathrm{a}$ & $\mathrm{b}$ & $\mathrm{c}$ \\
\hline $\mathrm{h}$ & & $\mathrm{d}$ \\
\hline $\mathrm{g}$ & $\mathrm{f}$ & $\mathrm{e}$ \\
\hline
\end{tabular}

\section{Fig.3: Texture Unit Ordering}

A more detail review of texture unit demonstrate that the absent TU's involve 2's in their texture unit. This is the case when neighbors and central pixels have the same values. If there is a deficit of 2's then TU will take only $0,1,3$ and 4 which means that the available real number of different textures are 48 instead of 58, that is 65536 and 390625 the spectrum will be never totally covered, thus the power of the tactic is misused. It encounters on texture Unit number also. To overcome this, Rough texture is used in the proposed tactic.

Rough Texton is a way to illustrate the texture property in a way better than the Texton since Rough texton uses the Rough texture unit (RTU) instead of texture unit (TU). By including rough set concept to the texton will generates all ranges of the values including ' 2 ' in the RTU [23]. Then, it helps to generates distinct textures are 58 instead of 48 . It covers total spectrum which is not possible by texton only means without rough set concept. Using Rough Set techniques provide a more flexible way of assigning values to the TU boxes Fi.

It can also be mentioned that rough set theory [23], in other side to fuzzy set theory, evidently distinguishes two important concepts like vagueness and uncertainty, these two concepts are often confused. Vagueness of a set can be defined by approximations. uncertainty relates to the elements of a set, such that the properties of elements are to be explained by the rough membership function.

\subsection{Extraction of Texels}

After abstraction of fuzzy texton images need to extract the texels from them. The local properties [24] used to extract the feature vectors used here come under two categories: one is about color information and other is about texture. Some of the significant features of texture properties (cluster properties) are Local Homogeneity, Cluster Shade and Cluster Prominence.

Local homogeneity:

$$
\sum_{i, j=0}^{l} \frac{1}{1+(i-j)^{2}} a(i, j)
$$

Cluster shade:

$$
\sum_{i, j=0}^{l}\left(i-M_{r}+j-M_{s}\right)^{3} a(i, j)
$$

Cluster Prominence:

$$
\begin{array}{r}
\sum_{i, j=0}^{l}\left(i-M_{r}+j-M_{s}\right)^{4} a(i, j) \\
\text { where } M_{r}=\sum_{i, j=0}^{l} i B(i, j) \\
\text { and } M_{s}=\sum_{i, j=0}^{l} j B(i, j)
\end{array}
$$

There are three important properties regarding color information. They are Color Expectancy, Color Variance and Skewness.

\section{Color Expectancy:}

$$
F_{i}=\frac{1}{M} \sum_{j=1}^{M} B_{i j}
$$

Color Variance:

$$
\delta_{i}=\left(\frac{1}{M} \sum_{j=1}^{M}\left(B_{i j} F_{i}\right)^{2}\right)^{\frac{1}{2}}
$$

Skewness:

$$
\sigma_{i}=\left(\frac{1}{M} \sum_{j=1}^{M}\left(B_{i j} F_{i}\right)^{3}\right)^{\frac{1}{3}}
$$

\subsection{Classification of Images}

The proposed tactic uses the features of first and second order statistics into an entity for texton scrutinity. In order to improve the classification gain, the proposed tactic combines the features of first and second order statistics, instead of using them separately. Feature set leads to representation of the training and testing images. The absolute difference of the feature vector values of the query image and database images are also calculated. After that, in order to identify the relevant images, fixed threshold, K-NN classifier is used to measure the similarity between query image and the database images [25]. In case of fixed threshold, the threshold values are computed for different query images. The best threshold value is chosen as the threshold of that particular texture feature. The distance between these Feature Vectors helps in classifying the images into correct clusters.

\section{RESULTS AND DISCUSSION}

The proposed tactic is experimented with Brodatz, Vistex and Google color image databases, of size $512 \times 512$ original color texture images. The proposed Tactic is compared with Steerable Pyramid Decomposition [26] and Gabor Wavelets [27] tactics and produces the better results as shown in Table 1

Table 1. Mean classification rates for the two different texture image datasets using K-NN classifier

\begin{tabular}{|l|l|l|l|}
\hline $\begin{array}{c}\text { Image } \\
\text { Dataset }\end{array}$ & $\begin{array}{c}\text { Steerable } \\
\text { Pyramid } \\
\text { Decomposition }\end{array}$ & $\begin{array}{c}\text { Gabor } \\
\text { Wavelets }\end{array}$ & $\begin{array}{c}\text { Proposed } \\
\text { Tactic }\end{array}$ \\
\hline Brodatz & $89.57 \%$ & $89.57 \%$ & $90.89 \%$ \\
\hline VisTex & $88.43 \%$ & $89.78 \%$ & $91.35 \%$ \\
\hline Google & $82.67 \%$ & $87.63 \%$ & $92.86 \%$ \\
\hline
\end{tabular}


Figure 4 shows the comparison graph of mean classification rates for the three databases Brodatz, VisTex and Google.

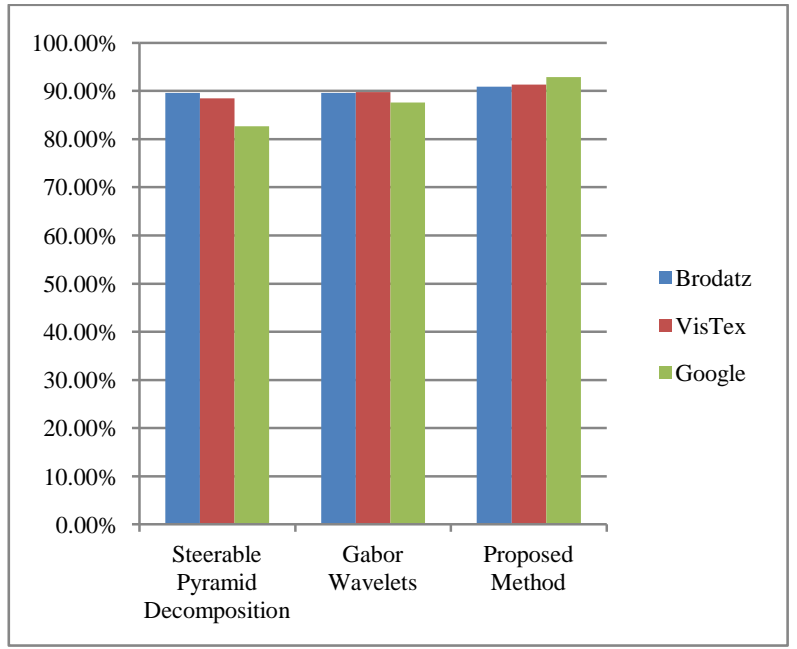

Fig.4: Comparison of Mean classification rates for three databases Brodatz, VisTex and Google.

\section{REFERENCES}

[1] Texture scrutinity. In: Chen, C.H; Pau, L.F. \& Wang, P.S.P., (eds). The handbook of pattern recognition and computer vision. 2nd ed. World Scientific Publishing Co., ISBN 9-810-23071-0, Singapore.

[2] Texture scrutinity in machine vision, World Scientific Publishing, 981-02-4373-1, Singapore.

[3] Handbook of texture scrutinity, Imperial College Press, 1-84816-115-8, UK.

[4] Doi K: Computer-aided diagnosis in medical imaging: Historical review, current status and future potential. Comput Med Imaging Graph 31(4-5):198-211, 2007

[5] Zaidi H, Vees H, Wissmeyer M: Molecular PET/CT imaging guided radiation therapy treatment planning. Acad Radiol 16(9):1108-33, 2009

[6] Marcus C, Ladam-Marcus V, Cucu C, Bouché O, Lucas $\mathrm{L}$, Hoeffel $\mathrm{C}$ : Imaging techniques to evaluate the response to treatment in oncology: Current standards and perspectives. Crit Rev Oncol/ Hematol 72(3):217-38, 2009

[7] M. Tuceryan, A.K. Jain, Texture scrutinity, in: C.H. Chen, L.F. Pau, P.S.P. Wang (Eds.), Handbook of Pattern Recognition and Computer Vision, 1993.

[8] A.R. Rao, A Taxonomy for Texture Description and Identi0cation, Springer, Berlin, 1990.

[9] F. Tomita, S. Tsuji, Computer Scrutinity of Visual Textures, Kluwer Academic, Hingham, MA, 1990.

[10] T.N. Tan, Geometric transform invariant texture scrutinity.

[11] R.M. Haralick, Statistical and structural approaches to Texture, Proc.

[12] T.N. Tan, Texture segmentation approaches: a brief review, Proceedings of CIE and IEEE International
Conference on Neural Networks and Signal Processing, Guangzhou, China, November 1993.

[13] T.R. Reed, J.M.H. Du Buf, A review of recent texture segmentation and feature extraction techniques.

[14] L. Van Gool, P. Dewaele, A. Oosterlinck, Survey: texture scrutinity anno 1983

[15] R. Chellappa, R.L. Kashyap, B.S. Manjunath, Portrait based texture segmentation and classi0cation, in: C.H. Chen, L.F. Pau, P.S.P. Wang (Eds.), Handbook of Pattern Recognition and Computer Vision, 1993.

[16] B. Julesz, T. Caelli, On the limits of fourier decompositions in visual texture perception.

[17] B. Julesz, Experiments in the visual perception of texture, Sci. Am

[18] Ojala, T. and M Pietikäinen: Texture Classification. Machine Vision and Media Processing Unit, University of Oulu, Finland. Available at http://homepages.inf.ed.ac.uk/rbf/CVonline/LOCAL_CO PIES/OJALA1/ texclas.htm. January, 2004.

[19] F. Cohen et al., Classi0cation of rotated and scaled texture images using Gaussian Markov random 0eld portraits, IEEE Trans.

[20] K. Sivakumar, Morphologically constrained GRFs: applications to texture synthesis and scrutinity, IEEE Trans.

[21] Fang Liu, R.W. Picard, Periodicity, directionality, and randomness: wold features of image and portraiting and retrieval, IEEE Trans. Pattern Anal. Mach. Intell.

[22] Fang Liu, R.W. Picard, Periodicity, directionality, and randomness: wold features for perceptional pattern recognition, Proceedings of the International Conference on Pattern Recognition, Vol. 11, Jerusalem, October 1994.

[23] Aboul Ella Hassanien, Ajith Abraham, James F. Peters, Gerald Schaefer, Christopher Henry, "Rough Sets and Near Sets in Medical Imaging: A Review", IEEE trans. on information technology in biomedicine, 2009.

[24] S Putheti, SR Edara, SA Edara, "CBIR using Texels of colour Fuzzy Textons" , Hybrid Intelligent Systems (HIS), 2012 12th International Conference on, 461-467, 2012.

[25] E. Mirkes, KNN and Potential Energy (Applet) University of Leicester. Available: http: //www.math.le.ac.uk/people/ag153/homepage/KNN/ KNN3.html, 2011

[26] Simoncelli E.P., Freeman W.T, “The steerable pyramid: A flexible architecture for multi-scale derivative computation," Proceedings of IEEE ICIP 13, pp:891906,1995

[27] Arivazhagan S., Ganesan L., Priyal S.P, "Texture classification using gabor wavelets based rotation invariant features," Pattern Recognition Letters, vol.27, pp:1976-1982, 2006 